\title{
Estimação de tendência genética para produção de leite na raça Holandesa no estado de Minas Gerais
}

\author{
[Estimate of genetic trend for milk production in Holstein in the State of Minas Gerais] \\ W.J. Ferreira ${ }^{1}$, N.M. Teixeira ${ }^{2,5}$, R.A. Torres ${ }^{3}$, R.B. Barra ${ }^{4}$ \\ ${ }^{1}$ Faculdade do Noroeste de Minas (FINOM) \\ Caixa Postal 201 \\ 38600-000 - Paracatu, MG \\ ${ }^{2}$ Embrapa Gado de Leite - Juiz de Fora, MG \\ ${ }^{3}$ Departamento de Zootecnia - UFV - Viçosa, MG \\ ${ }^{4}$ Associação de Criadores de Gado Holandês de Minas Gerais - Juiz de Fora, MG \\ ${ }^{5}$ Bolsista do CNPq
}

\begin{abstract}
RESUMO
Utilizaram-se 75.567 registros de produção de leite, de 40.941 vacas da raça Holandesa, distribuídos em 561 rebanhos e obtidos no período de 1980 a 2002. Valores genéticos de vacas e touros, obtidos por meio de modelo animal, foram usados para estimação de tendências genéticas, considerando-se as quatro trilhas de seleção conectando duas gerações. Estimativas de componentes de variância e valores genéticos previstos foram obtidos por meio do sistema MTDFREML. O modelo de análise incluiu os efeitos fixos de rebanho-ano-estação de parto, grupo genético, idade da vaca ao parto como covariável (efeitos linear e quadrático) e os efeitos aleatórios de animal, permanente de meio e erro. O maior valor de ganho genético anual, $57,7 \mathrm{~kg} / \mathrm{ano}$, foi para a trilha touros pais de touros no período de 1986 a 1991. Para a trilha vacas mães de touros, os valores foram negativos e para vacas mães de vacas passou de negativo de 1970 a 1985 para 16,9kg/ano, de 1986 a 1999 . Quando as trilhas de seleção foram combinadas, o ganho genético anual estimado no período 1970 a 1999 foi $6,71 \pm 3,78 \mathrm{~kg}$, correspondendo a $0,1 \%$ da média. As estimativas de tendência genética obtidas estão muito aquém do teoricamente possível.
\end{abstract}

Palavras-chave: gado de leite, ganho genético, método REML, modelo animal

\begin{abstract}
Production records from 75,567 lactations of 40,941 Holstein cows in 561 herds from 1980 to 2002 were used. Predicted cow and sire breeding values from an animal model were used to estimate genetic trend considering the four selection paths connecting two generations. The variance component estimates and breeding value predictions were obtained using the MTDFREML program package. The model of analysis accounted for the fixed effects of herd-year-season of calving, genetic group, age of calving as covariate (linear and quadratic) and the random effects of animal, permanent environment and error. The greatest annual genetic gain estimate was 57.7kg/yr for the path of sires of bulls from 1986 to 1991. For the path of dams of bulls, estimates were negative and for dams of cows changed from negative from 1970 to 1985 to $16.9 \mathrm{~kg} / \mathrm{yr}$, from 1986 to 1999 . When all paths of selection were combined, estimated annual genetic gain from 1970 to 1999 was $6.71 \pm 3.78 \mathrm{~kg}$, corresponding to $0.1 \%$ of the average production. Estimates of genetic trend were much less than theoretically possible.
\end{abstract}

Keywords: dairy cattle, genetic trend, REML, animal model

Recebido em 9 de junho de 2004

Aceito em 28 de março de 2006

Endereço (mailing address):

Rodovia MG 188 KM 167, s/nº, Bairro Fazendinha, Paracatu, MG

E-mail: ferreirawj@uol.com.br 


\section{INTRODUÇÃO}

Desde a década de 50, em vários países, vêm sendo divulgadas estimativas de ganhos genéticos anuais ou tendências genéticas, empregando-se diferentes procedimentos de estimação. Os valores encontrados, em geral, variam com o procedimento. Tendência genética pode ser estimada por meio da regressão dos valores genéticos sobre o período (Hintz et al., 1978; Freitas et al., 1995; Durães et al., 2001), regressão da produção sobre o período (Teixeira et al., 1994) ou por meio de diferencial de seleção (Van Tassel e Van Vleck, 1991; Silva et al., 2001).

Teixeira et al. (1994) estimaram tendência genética na produção de leite da raça Holandesa, no estado do Paraná, no período de 1980 a 1988. Utilizaram 8.882 primeiras lactações da filhas de 143 touros, pertencentes a 79 rebanhos e dois métodos para estimar a tendência genética. $\mathrm{O}$ método I baseou-se em um procedimento de regressão ponderada das constantes de quadrados-mínimos para anos sobre anos. O modelo para produção na primeira lactação, corrigida para idade e 305 dias, incluiu os efeitos fixos de rebanho, ano, estação (modelo A), mais $o$ efeito fixo de touro dentro de rebanho (modelo B). A tendência genética estimada, dobrando-se a diferença entre os coefícientes obtidos pelos modelos B e A, foi de $128 \mathrm{~kg}$. No método II, as estimativas para tendência genética foram obtidas como mudança nos méritos genéticos dos touros com valores de $139 \mathrm{~kg}$, quando o valor genético do touro foi usado no ano de parto da sua primeira filha, e de $94 \mathrm{~kg}$, quando foi usado no ano em que teve maior número de filhas.

Costa et al. (1999), ao utilizarem dados de 29.413 vacas da raça Holandesa, no Brasil, estimaram tendências genéticas no período de 1977 a 1990, com um modelo de touro e análise bivariada. As estimativas de ganho genético anual não-ponderado e ponderado, pelo número de filhas de touros pais de vacas, foram 24,0 e $33,0 \mathrm{~kg} / \mathrm{ano}$ de leite e 0,64 e $0,85 \mathrm{~kg} / \mathrm{ano}$ de gordura respectivamente, para vacas nascidas entre 1977 e 1990.

De acordo com Hansen (2000), a tendência genética para produção de leite na raça Holandesa, nos Estados Unidos, aumentou consideravelmente ao longo dos anos. A tendência genética média foi $37 \mathrm{~kg}$ durante a década de $60,79 \mathrm{~kg}$ durante a década de 70 , $102 \mathrm{~kg}$ na década de 80 e $116 \mathrm{~kg}$ de 1990 a 1996.

Abdallah e McDaniel (2000a) obtiveram ganho genético anual de $80,9 \mathrm{~kg}$ de leite, em um estudo utilizando registros de produção de animais da raça Holandesa, nos Estados Unidos, no período de 1971 a 1993. Também Abdallah e McDaniel (2000b), ao trabalharem com 23.052 registros de produção de leite de 8.575 vacas, nascidas no período de 1950 a 1993, com até seis partos, filhas de 681 touros, pertencentes a cinco rebanhos, nos Estados Unidos, observaram ganhos genéticos anuais de $-2,5,27,9,36,7 \mathrm{e}$ $94,7 \mathrm{~kg}$ nos períodos de 1950 a 1960,1960 a 1970, 1970 a 1980 e 1980 a 1993, respectivamente.

Silva et al. (2001) estimaram tendências genéticas para as diferentes trilhas de progresso, em dois períodos, para o ecótipo Mantiqueira da raça Holandesa, usando dados das cinco primeiras lactações de 1.406 vacas, filhas de 113 reprodutores, com partos entre 1952 e 1997, no estado de São Paulo. Tendências genéticas obtidas para produção de gordura, duração da lactação e período de serviço foram inexpressivas, sugerindo ter havido seleção somente para produção de leite.

Avaliação e seleção de touros por meio de teste de progênie são importantes para o progresso genético em gado de leite. Como não há teste de progênie para a raça Holandesa no Brasil, o país depende da importação de sêmen de touros melhorados, principalmente dos Estados Unidos e Canadá

O objetivo deste trabalho foi estimar tendências genéticas para produção de leite na raça Holandesa por meio de regressão de valores genéticos sobre ano de nascimento dos animais, considerando as trilhas de seleção propostas por Rendel e Robertson (1950).

\section{MATERIAL E MÉTODOS}

Utilizaram-se 49.927 registros de produção até a quinta lactação de vacas com partos ocorridos entre 1980 e 2002, filhas de 889 touros, de 360 rebanhos, provenientes do Serviço de Controle Leiteiro da Associação dos Criadores de Gado 
Holandês de Minas Gerais. Foram consideradas as lactações com encerramento normal, com duração entre 150 a 450 dias e com produções de leite superiores a $1.000 \mathrm{~kg}$ ou inferiores a $13.000 \mathrm{~kg}$. Para a estimação de componentes de variância, de parâmetros genéticos e de valores genéticos, utilizou-se modelo com os efeitos de rebanho-ano-estação de parto, grupo genético (puro de origem (PO) e $\geq 31 / 32$ de sangue Holandês), idade da vaca ao parto (linear e quadrático) e os efeitos aleatório de animal, permanente de ambiente e erro. As estações de parição foram: águas (de outubro a março) e seca (de abril a setembro). Os limites de idade dentro de ordem de parto foram: ordem de parto 1 ( $20 \mathrm{a}$ 60 meses), 2 (30 a 78 meses), 3 (41 a 96 meses), 4 (54 a 116 meses) e 5 (66 a 134 meses).

Para obtenção do arquivo de pedigree, utilizou-se o arquivo de cadastro de vacas, contendo a identificação da vaca, do pai e da mãe, com 65.783 animais, e o arquivo de cadastro de touros, contendo a identificação do touro, do pai e da mãe, com 5.894 animais. Os touros deveriam ter, pelo menos, cinco filhas e cada classe de rebanho-ano-estação de parto, no mínimo cinco registros de produção de filhas de, pelo menos, dois touros. O modelo usado foi: $\mathrm{y}=\mathrm{X} \beta+\mathrm{Zu}+\mathrm{Wpe}+\varepsilon$, em que:

$\mathrm{y}=$ vetor de produção de leite até 305 dias de lactação; $\mathrm{X}=$ matriz de incidência para os efeitos fixos de rebanho-ano-estação de parto, grupo genético e coeficientes de regressão linear e quadrático da produção sobre a idade da vaca ao parto; $\mathrm{Z}=$ matriz para efeito de animal; $\mathrm{W}=$ matriz para efeito permanente de ambiente; $\beta=$ vetor de efeitos fixos; $\mathrm{u}=$ vetor de efeitos aleatórios de animal; pe $=$ vetor de efeitos aleatórios permanentes de ambiente, nãorelacionados com os efeitos de $u$; e $\varepsilon=$ vetor de erros aleatórios.

$\mathrm{O}$ vetor $\mathrm{u}$ inclui somente o efeito aleatório de animal, conseqüentemente, os efeitos genéticos não aditivos são incluídos no termo pe.

Admite-se que os efeitos permanentes de ambiente e do erro sejam independentemente distribuídos, com média zero e variância $\sigma_{\mathrm{pe}}^{2} \mathrm{e}$ $\sigma_{\mathrm{e}}^{2}$, respectivamente, e não correlacionados com o efeito de animal u. Além disso,

$$
\begin{aligned}
& \operatorname{var}(\mathrm{u})=A \sigma_{\mathrm{a}}^{2}, \\
& \operatorname{var}(\mathrm{pe})=\mathrm{I} \sigma_{\mathrm{pe}}^{2}, \\
& \operatorname{var}(\varepsilon)=\mathrm{I} \sigma_{\mathrm{e}}^{2}=\mathrm{R}, \\
& \operatorname{var}(\mathrm{y})=\mathrm{ZAZ} \mathrm{Z}_{\mathrm{a}}^{2}+\mathrm{WI} \sigma_{\mathrm{pe}}^{2} \mathrm{~W}^{\prime}+\mathrm{R} .
\end{aligned}
$$

As equações de modelos mistos (EMM), para o melhor estimador linear não-viesado (BLUE) das funções estimáveis de $\beta$ e para o melhor preditor linear não-viesado (BLUP) de u e pe, são dadas pela solução do sistema seguinte:

$$
\left[\begin{array}{ccc}
X^{\prime} X & X^{\prime} Z & X^{\prime} W \\
Z^{\prime} W & Z^{\prime} Z+A^{-1} \alpha_{1} & Z^{\prime} W \\
W^{\prime} X & W^{\prime} Z & W^{\prime} W+I \alpha_{2}
\end{array}\right]\left[\begin{array}{c}
\beta \\
\hat{u} \\
\hat{p e}
\end{array}\right]=\left[\begin{array}{c}
x^{\prime} y \\
z^{\prime} y \\
w^{\prime} y
\end{array}\right]
$$

em que: $\quad \alpha_{1}=\sigma_{\mathrm{e}}^{2} / \sigma_{\mathrm{a}}^{2} ; \quad \alpha_{2}=\sigma_{\mathrm{e}}^{2} / \sigma_{\mathrm{pe}}^{2} ; \quad$ e $\mathrm{A}=$ matriz contendo os coeficientes de parentesco de Wright entre os animais.

Os componentes de variância, necessários à estimação dos parâmetros genéticos e dos valores genéticos dos animais, foram obtidos pelo método da máxima verossimilhança restrita (REML), utilizando-se o sistema multiple trait derivative-free restricted maximum likelihood (MTDFREML) desenvolvido por Boldman et al. (1995).

As médias dos valores genéticos foram calculadas por ano de nascimento dos animais, em cada trilha de seleção (Rendel e Robertson, 1950): touros pais de touros (SB), touros pais de vacas (SC), vacas mães de touros (DB) e vacas mães de vacas (DC). Para touros, foram calculadas duas médias por trilha de seleção: ponderada ou não pelo número de progênies.

O ganho genético realizado para cada trilha de seleção foi estimado por meio de regressão das médias dos valores genéticos sobre o ano de nascimento. $O$ ganho genético anual $(\Delta G)$ foi calculado pelo coeficiente de regressão dos valores genéticos sobre o ano de nascimento, para cada trilha de seleção, conforme proposto por Van Tassell e Van Vleck (1991):

$$
\Delta \mathrm{g}=1 / 4\left(\mathrm{~b}_{\mathrm{G}_{\mathrm{SB}} \cdot \mathrm{T}}+\mathrm{b}_{\mathrm{G}_{\mathrm{DB}} \cdot \mathrm{T}}+\mathrm{b}_{\mathrm{G}_{\mathrm{SC}} \cdot \mathrm{T}}+\mathrm{b}_{\mathrm{G}_{\mathrm{DC}} \cdot \mathrm{T}}\right) .
$$


O erro-padrão, associado à estimativa de ganho genético anual, foi obtido por meio da média dos erros-padrão dos coeficientes de regressão de cada trilha, conforme sugerido por Nizamani e Berger (1996).

\section{RESULTADOS E DISCUSSÃO}

As médias dos valores genéticos para produção de leite até 305 dias de lactação (P305), por ano de nascimento dos SB, DB, SC e DC, encontram-se representadas nas Fig. 1, 2, 3 e 4, juntamente com os valores genéticos médios do grupo base não selecionado, isto é, todas as vacas nascidas durante o mesmo ano. Na Tab. 1 encontram-se os ganhos genéticos anuais para cada trilha de seleção em função de períodos de tempo e para todas as vacas. Exceto para trilha $\mathrm{SC}$, os valores estimados dos coeficientes de determinação para as regressões para as outras trilhas foram maiores no período mais recente. A estimativa de ganho genético anual e o respectivo erro-padrão, considerando-se as quatro trilhas de seleção, foram de $6,71 \pm 3,78 \mathrm{~kg}$, correspondendo a $0,1 \%$ da média. Este valor é muito inferior aos relatados na literatura para a raça Holandesa (Abdallah e McDaniel, 2000a; Abdallah e McDaniel, 2000b; Hansen, 2000). Esses, também, foram inferiores aos ganhos genéticos encontrados no Brasil para a raça Holandesa, por Costa et al. (1999) e Durães et al. (2001). Segundo Rendel e Robertson (1950), são possíveis ganhos genéticos anuais para produção de leite de até $2 \%$ da média.

As médias anuais dos valores genéticos, ponderadas e não-ponderadas pelo número de filhos, para a trilha SB (Fig. 1) foram, em geral, semelhantes, o que pode ser explicado pelo fato de que quase todos os reprodutores tinham filhos que foram utilizados como touros nos rebanhos. Verificou-se tendência de melhoria dos valores genéticos ao longo dos anos. Contudo, as médias dos valores só foram maiores do que as da população-base no período de 1978 a 1983 e após 1989.

Tabela 1. Estimativas de mudança genética anual (b) para produção de leite para cada trilha de seleção e todas as vacas com respectivos erros-padrão (EP) e coeficientes de determinação $\left(\mathrm{R}^{2}\right)$

\begin{tabular}{lccccccc}
\hline \multirow{2}{*}{$\begin{array}{l}\text { Trilha de } \\
\text { seleção }\end{array}$} & \multirow{2}{*}{ Período } & \multicolumn{3}{c}{ Ponderado } & \multicolumn{3}{c}{ Não-ponderado } \\
\cline { 2 - 8 } SB & $1973-1985$ & $\mathrm{~b}$ & $\mathrm{EP}$ & $\mathrm{R}^{2}$ & $\mathrm{~b}$ & $\mathrm{EP}$ & $\mathrm{R}^{2}$ \\
& $1986-1991$ & 57,7 & 14,5 & 0,31 & 18,3 & 10,9 & 0,22 \\
& $1973-1991$ & 12,6 & 17,0 & 0,74 & 57,7 & 17,0 & 0,74 \\
DB & $1970-1985$ & - & -2 & 0,10 & 8,7 & 6,6 & 0,10 \\
& $1986-1988$ & - & - & - & $-8,9$ & 7,5 & 0,12 \\
& $1970-1988$ & - & - & - & $-87,7$ & 46,7 & 0,78 \\
SC & $1970-1985$ & 15,6 & 16,9 & 0,06 & $-4,7$ & 5,5 & 0,05 \\
& $1986-1995$ & 4,2 & 18,4 & 0,01 & 13,7 & 5,9 & 0,01 \\
& $1970-1995$ & 28,1 & 7,0 & 0,40 & 10,5 & 2,4 & 0,41 \\
DC & $1970-1985$ & - & - & - & $-3,4$ & 3,5 & 0,44 \\
& $1986-1999$ & - & - & - & 16,9 & 2,4 & 0,81 \\
& $1970-1999$ & - & - & - & 11,9 & 1,6 & 0,68 \\
\multirow{2}{*}{ Todas as } & $1970-1985$ & - & - & - & $-4,0$ & 2,6 & 0,15 \\
vacas & $1986-1999$ & - & - & - & 19,1 & 2,0 & 0,89 \\
& $1970-1999$ & - & - & - & 15,1 & 1,5 & 0,79 \\
\hline
\end{tabular}

SB: touros pais de touros; SC: touros pais de vacas; DB: vacas mães de touros; DC: vacas mães de vacas. 


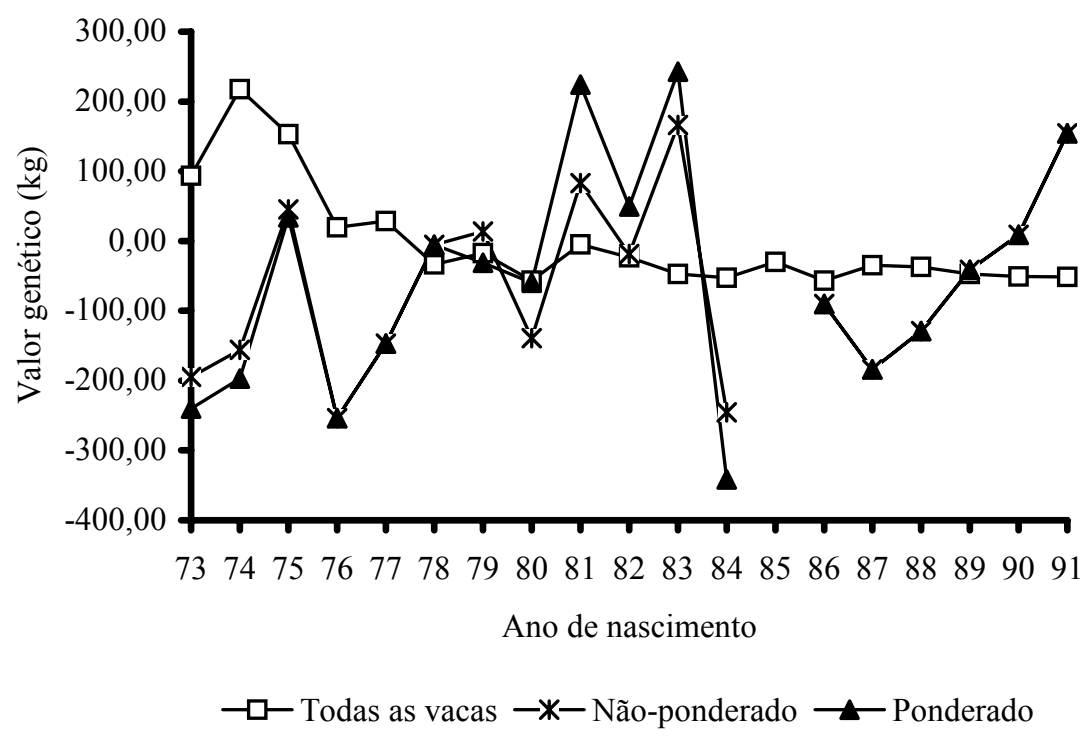

Figura 1. Médias dos valores genéticos para produção de leite até 305 dias de lactação dos touros pais de touros, ponderadas e não-ponderadas pelo número de filhos, de acordo com o ano de nascimento dos touros, e médias dos valores genéticos de todas as vacas nascidas no mesmo ano.

A trilha SB foi a que apresentou as maiores estimativas de ganho genético anual, com as médias ponderadas de, respectivamente, 30,9, 57,7 e $12,6 \mathrm{~kg}$, nos períodos de 1975 a 1985 , 1986 a 1991 e total.

Com exceção de 1984, 1986 e 1987, as médias dos valores genéticos das DB nos demais anos foram menores do que a média de todas as vacas nascidas no mesmo ano (Fig. 2). As estimativas de tendência genética (Tab. 1) foram negativas, indicando que não houve seleção nessa trilha. Possivelmente, a inclusão de características não produtivas, como critério de seleção, foi determinante para obtenção desses resultados. Além disso, podem ter sido devido a baixa intensidade de seleção, uma vez que a maioria das vacas permaneceram nos rebanhos para serem mães da próxima geração.

As médias anuais dos valores genéticos para produção de leite até 305 dias de lactação, na trilha de seleção SC, são representadas na Fig. 3. De 1973 a 1984, com exceção de 1978, as médias de valores genéticos dos touros dessa trilha foram menores do que as médias dos valores genéticos da população-base não selecionada, com ganho genético muito pequeno. A seleção dos animais nessa trilha passou a ocorrer a partir de 1985, quando, provavelmente, a produção de leite passou a ser considerada como característica principal na seleção dos animais, promovendo aumento nas médias dos valores genéticos dos animais escolhidos para reprodução.

Também a partir de 1985, as médias ponderadas foram maiores do que as não-ponderadas, possivelmente por causa da escolha dos melhores animais para pais das vacas futuras.

As médias anuais dos valores genéticos para a trilha DC são apresentadas na Fig. 4. Na maioria dos anos, as médias de valores genéticos foram maiores do que as médias dos valores genéticos da população-base não selecionada, evidenciando a seleção das melhores vacas para serem mães das futuras vacas dos rebanhos. Entretanto, a seleção foi menos intensa do que nas demais trilhas, como esperado, devido à baixa taxa reprodutiva das vacas. É importante destacar que, na maioria das vezes, o descarte de 
vacas dos rebanhos é realizado em decorrência de doenças ou por outras razões diferentes da baixa produção. Dessa forma, há aumento no número de vacas nos rebanhos, o que torna disponível mais animais para reprodução. Resultados similares foram descritos por Silva et al. (2001).
Ganhos genéticos obtidos por meio de seleção de vacas foram também relatados por Abdallah e McDaniel (2000b), quando avaliaram produções de vacas da raça Holandesa, pertencentes a rebanhos leiteiros experimentais, nos Estados Unidos.

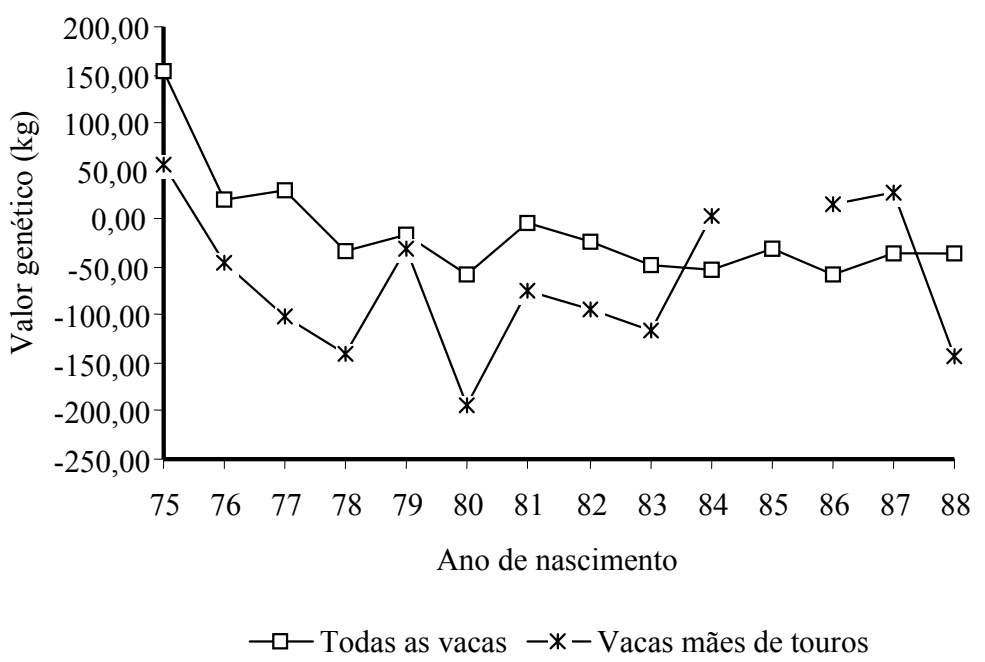

Figura 2. Médias dos valores genéticos para produção de leite até 305 dias de lactação das vacas mães de touros, de acordo com o ano de nascimento das vacas, e médias dos valores genéticos de todas as vacas nascidas no mesmo ano.

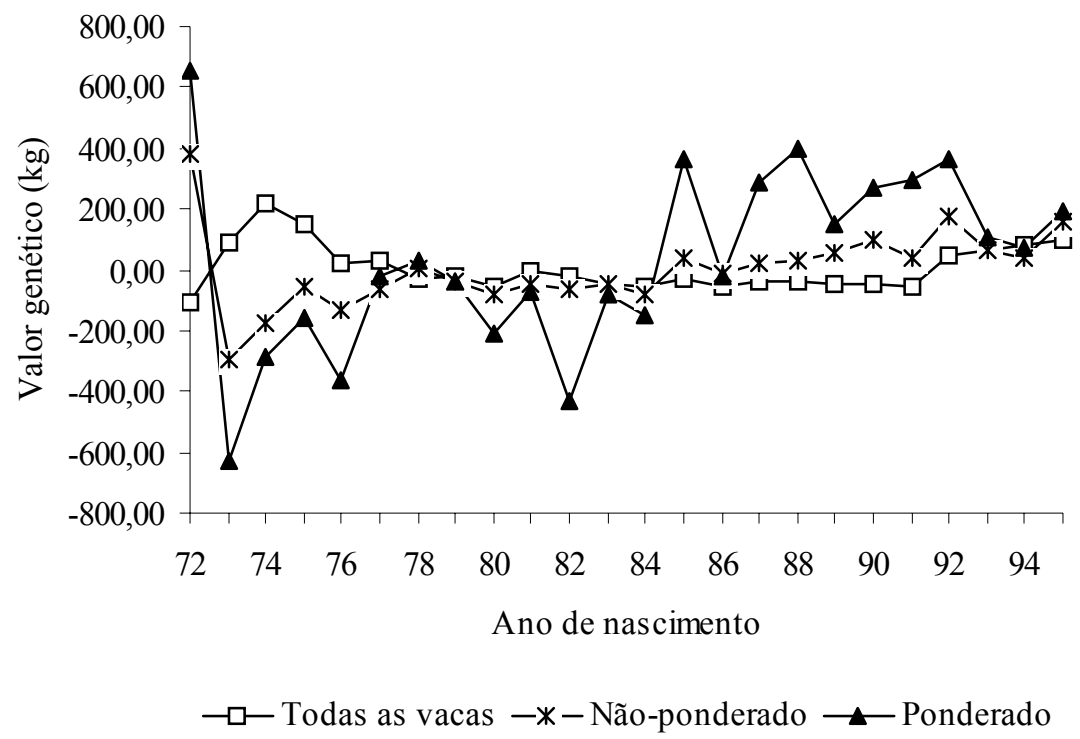

Figura 3. Médias dos valores genéticos para produção de leite até 305 dias de lactação dos touros pais de vacas, ponderadas e não-ponderadas pelo número de filhas, de acordo com o ano de nascimento dos touros, e médias dos valores genéticos de todas as vacas nascidas no mesmo ano. 


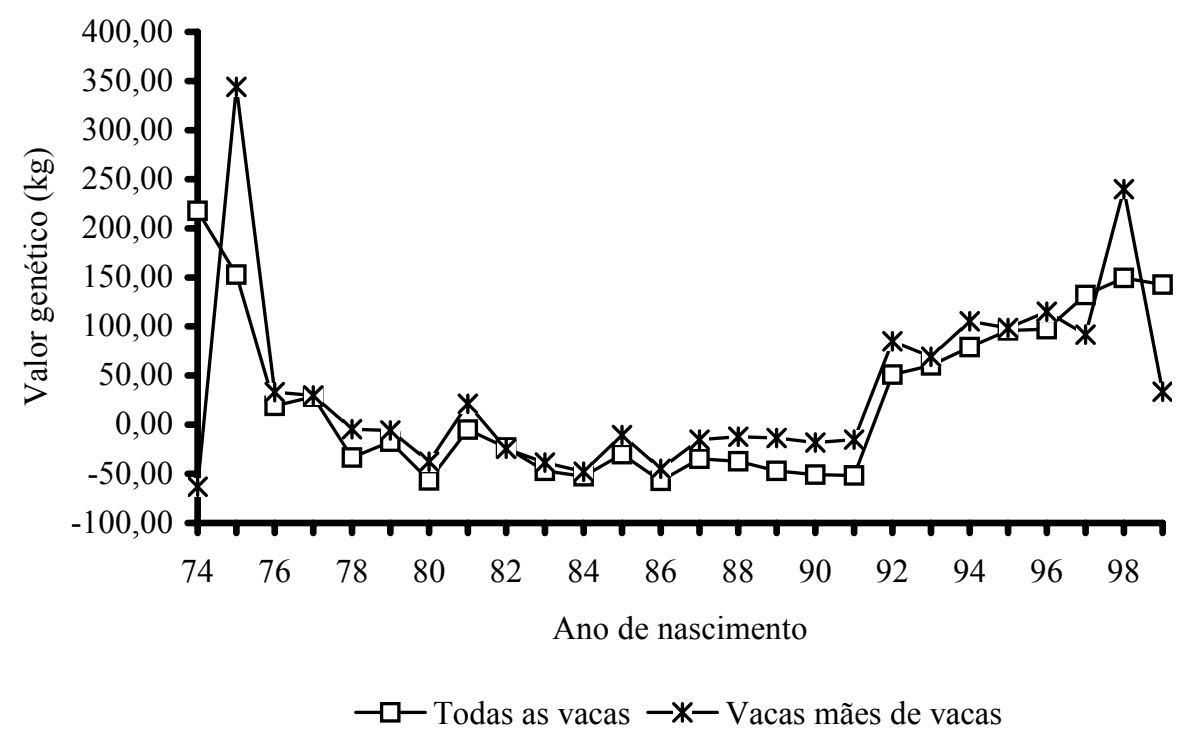

Figura 4. Médias dos valores genéticos para produção de leite até 305 dias de lactação das vacas mães de vacas, de acordo com o ano de nascimento das vacas, e médias dos valores genéticos de todas as vacas nascidas no mesmo ano.

Burnside et al. (1992) e Nizamani e Berger (1996) encontraram maiores taxas de aumento dos valores genéticos dos pais do que das mães. Seleção mais intensa de pais de touros possibilita aos criadores maior aumento dos valores genéticos de seus animais ao longo do tempo.

O ganho genético na população total de vacas no período de 1986 a 1999, estimado por meio de regressão ponderada das médias dos valores genéticos das vacas sobre ano de nascimento, foi de $19,1 \mathrm{~kg} / \mathrm{ano}$, semelhante aos valores encontrados por Durães et al. (2001) ao usarem o mesmo procedimento de estimação.

\section{CONCLUSÕES}

As estimativas de tendência genética obtidas estão muito aquém do teoricamente possível, sugerindo que as práticas de seleção no período não foram adequadas. Possivelmente, a ênfase para características diferentes da produção de leite tenha contribuído para os baixos valores encontrados. Além disso, em razão da inexistência de teste de touros jovens na população, a seleção de pais e mães de touro foi pouco enfatizada.

\section{REFERÊNCIAS BIBLIOGRÁFICAS}

ABDALLAH, J.M.; McDANIEL, B.T. Genetic change in milk, fat, days open, and body weight after calving based on three methods of sire selection. J. Dairy Sci., v.83, p.1359-1363, 2000a.

ABDALLAH, J.M.; McDANIEL, B.T. Genetic parameters and trends of milk, fat, days open, and body weight after calving in North Carolina experimental herdes. J. Dairy Sci., v.83, p.13641370, 2000b.

BOLDMAN, K.G.; KRIESE, L.A.; VAN VLECK, L.D. et al. A manual for use of $M T D F R E M L$. A set of programs to obtain estimates of variances and covariances [DRAFT]. Beltsville: U.S. Department of Agriculture, Agricultural Research Service, 1995.

BURNSIDE, E.B.; JANSEN, G.B.; CIVATI, G. et al. Observed and theoretical genetic trends in a large dairy population under intensive selection. J. Dairy Sci., v.75, p. 2242-2253, 1992. 
COSTA, C.N.; BLAKE, R.W.; POLLAK, E.J. et al. Tendências genéticas das produções de leite e de gordura na raça Holandesa no Brasil. In: REUNIÃO ANUAL DA SOCIEDADE BRASILEIRA DE ZOOTECNIA, 36., 1999, Porto Alegre-RS. Anais... Porto Alegre: SBZ, 1999. p. 131.

DURÃES, M.C.; FREITAS, A.F.; VALENTE, J. et al. Tendência genética para a produção de leite e de gordura em rebanhos da raça Holandesa no Estado de Minas Gerais. Rev. Bras. Zootec., v.30, p.66-70, 2001.

FREITAS, A.F.; VERNEQUE, R.S.; TEIXEIRA, N.M. et al. Estimativas de tendências genéticas em gado Holandês do Estado de Minas Gerais. In: REUNIÃO ANUAL DA SOCIEDADE BRASILEIRA DE ZOOTECNIA, 32., 1995, Brasília-DF. Anais... Brasília: SBZ, 1995. p. 660.

HANSEN, L.B. Symposium: selection for milk yield - Consequences of selection for milk yield from a geneticist's viewpoint. J. Dairy Sci., v.83, p.1145-1150, 2000.

HINTZ, R.L.; EVERETT, R.W.; VAN VLECK, L.D. Estimation of genetic trends from cow and sire evaluations. J. Dairy Sci., v.61, p.607-613, 1978.

NIZAMANI, A.H.; BERGER, P.J. Estimates of genetic trend for yield traits of the registred Jersey population. J. Dairy Sci., v.79, p.487-494, 1996.

RENDEL, J.M.; ROBERTSON, A. Estimation of genetic gain in milk yield by selection in a closed herd of dairy cattle. J. Gen., v.50, p.1-8, 1950.

SILVA, M.V.G.B.; FERREIRA, W.J.; COBUCI, J.A. et al. Estimativas de tendência genética para características produtivas em um rebanho do ecótipo Mantiqueira. Rev. Bras. Zootec., v.30, p.1466-1475, 2001.

TEIXEIRA, N.M.; FREITAS, A.F.; RIBAS, N.P. et al. Tendências genéticas em rebanhos da raça Holandesa no Estado do Paraná. I. Produção de leite. Rev. Bras. Zootec., v.23, p.983-991, 1994.

VAN TASSELL, C.P.; VAN VLECK, L.D. Estimates of genetic selection differentiais and generation intervals for four paths of selection. $J$. Dairy Sci., v.74, p.1078-1086, 1991. 